\title{
Esophageal anastomosis - how the granulation phase of wound healing improves the incidence of anastomotic leakage
}

\author{
RENATA TABOLA ${ }^{1}$, KATARZYNA AUGOFF ${ }^{1}$, ANDRZEJ LEWANDOWSKI ${ }^{1}$, PIOTR ZIOLKOWSKI ${ }^{2}$, \\ PIOTR SZELACHOWSKI ${ }^{1}$ and KRZYSZTOF GRABOWSKI ${ }^{1}$ \\ Departments of ${ }^{1}$ Gastrointestinal and General Surgery, and ${ }^{2}$ Pathomorphology, Wrocław Medical University, \\ 50-369 Wrocław, Poland
}

Received January 31, 2016; Accepted June 2, 2016

DOI: $10.3892 / \mathrm{ol} .2016 .4873$

\begin{abstract}
A two-stage esophagectomy with an interval for reconstruction of the esophagus creates an opportunity for the esophageal stump to recover from vessel injury and allows the formation of granulation tissue rich in proangiogenic factors, including transforming growth factor $\beta$ (TGF- $\beta$ ) and vascular endothelial growth factor A (VEGF-A), which may have an impact on anastomosis healing. The present study comprised 25 patients (27 in total, 2 succumbed to complications following surgery) who underwent two-stage esophagectomy for squamous cell carcinoma in the Department of Gastrointestinal and General Surgery, Wrocław Medical University (Wrocław, Poland) between January 2007 and December 2012. Immunohistochemical staining for VEGF-A and TGF- $\beta$ was performed to evaluate esophageal wall specimens at the time of esophagostomy construction and prior to anastomosis, in which the cervical esophagus was connected with the colon or ileum. At the time of reconstructive surgery, a significant increase in microvessel density was observed in all esophageal specimens $(\mathrm{P}<0.03)$. Significant differences were also identified in the immunohistochemical staining intensity of TGF- $\beta$ and VEGF-A in the epithelium of all esophageal specimens between biopsies obtained from normal esophageal tissues at the time of esophagectomy and during reconstructive surgery. Delayed anastomosis construction provides an advantage for the esophageal stump to accumulate proangiogenic growth factors, which overlap with the subsequent proliferative stage of the anastomosed tissue and thus supports its recovery, creating an optimal environment for the healing of any fistulas.
\end{abstract}

Correspondence to: Dr Renata Tabola, Department of Gastrointestinal and General Surgery, Wrocław Medical University, 66 Curie-Sklodowskiej, 50-369 Wrocław, Poland

E-mail: tabrena@op.pl

Key words: esophageal cancer, esophagectomy, esophageal reconstruction, angiogenesis, anastomosis healing

\section{Introduction}

Reconstruction of alimentary tract continuity following esophagectomy may be achieved in several ways. The reconstruction method is able to indirectly affect cancer survival and has an impact on postoperative dysphagia (1). For this purpose, the gastric tube or the large or small bowel may be mobilized and transposed through the anterior or posterior mediastinum. Each route of transposition has advantages, but are often under debate, since the posterior route is shorter than the anterior route, but mediastinal leakage is more dangerous than leakage on the neck. Anastomotic leakage is a feared complication of reconstruction with an incidence of 3-30\% and a mortality rate of $25-50 \%$ (2-7).

A gastric tube is most commonly used to aid reconstructive surgery (3). The anterior route of reconstruction omits the tumor bed and possible tumor recurrence does not affect the passage of food. Anastomosis of the esophageal stump is performed subcutaneously, which may have an impact on the possible care and course of the leakage. Anterior interposition of the cervical esophagus may lead to additional damage of its blood supply and encourage the leakage as a result of ischemic necrosis. The interval between esophagectomy and reconstructive surgery has an obvious disadvantage for the patient in that it excludes early oral feeding, but two-stage esophagectomy is a relatively safe form of surgery that may reduce the risk of critical complications in high risk patients $(8,9)$. Furthermore, in certain circumstances it creates the opportunity for the esophageal stump to recover from vessel injury and accumulate growth factors characteristic for the granulation stage of wound healing (10).

Vascular endothelial growth factor A (VEGF-A) and transforming growth factor $\beta$ (TGF- $\beta$ ) are important in angiogenesis occurring during the proliferation phase of wound healing. In normal tissue conditions, these factors are expressed at minimal levels. Mechanical injury and hypoxia provoke strong upregulation of VEGF-A expression, which then correlates temporarily and spatially with the growth of new blood vessels (10-12). VEGF is crucial for angiogenesis, controlling blood vessel formation and growth (13). VEGF and TGF modulate endothelial cell proliferation and are important for the creation of a favorable microenvironment for newly formed microvessels; these growth factors upregulate matrix 
metalloproteinases, which participate in the degradation of extracellular matrix (ECM) components, and support endothelial cell migration, invasion and survival (14-16). TGF- $\beta$ participates in all phases of wound healing, which includes proliferation, inflammation and ECM remodeling. The growth factor mediates fibroblast activation, regulates the expression of cytokines, including VEGF, and controls the synthesis of key ECM components, such as collagen I and III (17). The final step in the proliferation phase is the development of granulation tissue. With the progression of wound healing and formation of granulation tissue, proliferation and vascularization cease, collagen synthesis increases and the wound maintains a balance between ECM degradation and synthesis (18-20). Subsequently, scar tissue forms and the wound enters the remodeling stage, which lasts from several months to years (21). However, angiogenesis is not completely finished yet, and the tissue remains highly vascularized. Typical features of this stage include regression and maturation of the vascular structure and substitution of the granulation tissue (provisional ECM) into a permanent collagenous matrix, which guides the vessels into an optimally distributed and functioning network $(20,21)$. Collagen III, which is characteristic of granulation tissue, is now extensively replaced by collagen I (10,13-22).

\section{Patients and methods}

Study subjects. The present study comprised 27 patients out of 57 patients who underwent esophageal resection for esophageal or gastroesophageal cancer at the Department of Gastrointestinal and General Surgery, Wrocław Medical University (Wrocław, Poland) between January 2007 and December 2012. Eligible inclusion criteria included the two-stage procedure, histopathological tumor type (squamous cell carcinoma) and anterior coloplasty or ileo-coloplasty for reconstruction of the digestive tract. The remaining patients did not fit the eligibility criteria (20 of them had gastroesophageal cancer and 10 had reconstruction made of the gastric tube). Esophagectomy with two-field lymphadenectomy was followed by esophagostomy and gastrostomy. After a mean time of 3.3 months (range, 2-6 months) and additional radiotherapy and/or chemotherapy that lasted 1.8-3.6 months (mean, 2.5 months), patients underwent reconstructive surgery. The total dose of radiotherapy was 50-60 Gy over a 5-week period. Regimes of chemotherapy were mainly based on paclitaxel and cisplatin at a dosage of $150 \mathrm{mg} / \mathrm{m}^{2}$ and $50 \mathrm{mg} / \mathrm{m}^{2}$, respectively, every 14 days. The median number of chemotherapy cycles was 4 . Additionally, 12 out of the 27 eligible patients who were treated in the same department between January 2010 and December 2012 comprised a study group that was analyzed immunohistochemically. The remaining patients were evaluated retrospectively.

Baseline characteristics of the patients are presented in Table I. The patients were in poor general condition preoperatively. A total of 16 were in a state of malnutrition with a body mass index (BMI) of <17 (healthy range, 18.5-24.9), while 10 patients had underlying pulmonary insufficiency with a vital capacity or forced expiratory volume 1 of $\leq 50 \%$ predicted value (normal predicted value, $\geq 80 \%$ ). A total of 4 patients had chronic renal failure with blood creatinine levels between 1.5 and $2.5 \mathrm{mg} / \mathrm{dl}$ (reference value, $0.55-1.02 \mathrm{mg} / \mathrm{dl}$ ),
9 had uncontrolled diabetes with fasting blood glucose levels between 140-180 mg/dl (normal level range, 65-99 mg/dl), 8 had coronary ischemic disease, 2 of which had stents in the coronary arteries and 1 patient had an additional stent in the internal carotid artery, 19 patients were or used to be heavy smokers, and 8 had more than one comorbidity.

In all cases, a three-incision approach was applied (right thoracotomy, laparotomy and left cervical incision). Pyloroplasty was performed as a standard procedure following esophagectomy. All neck anastomoses were made in an end-side to end manner, with double-layered, interrupted suturing. Patients remained on gastrostomy following the reconstructive surgery, until water-soluble contrast swallow examination was routinely performed days 6-8 post-surgery, prior to the introduction of oral intake. In 4 patients, anastomotic leakage was observed. Anastomotic leakage was defined as discharge of saliva and or intestinal content through a wound on the neck or as an infected neck wound incision. The severity of the neck leakage was evaluated by contrast examination and, in selected cases, by endoscopy.

Postoperative mortality was defined as any mortality during hospital stay, irrespective of its length.

Microvessel density in the wall of the esophageal stump was investigated directly after esophagectomy and 2-6 months later, once the reconstruction had been performed.

Immunohistochemistry. Immunohistochemistry was performed to evaluate esophageal wall specimens at the time of esophagostomy construction and prior to anastomosis of the cervical esophagus with the colon or ileum. For immunohistochemical examination, formalin-fixed, paraffin-embedded tissue sections (3-5- $\mu \mathrm{m}$ thick) were deparaffinized in two changes of xylene for $5 \mathrm{~min}$ each. The sections were subsequently hydrated in decreasing concentrations of ethanol (96, 80 and 60\%) and rinsed in water. Antigen retrieval was routinely performed by incubation in $10 \mathrm{mmol} / 1$ sodium citrate buffer ( $\mathrm{pH}$ 6.0) and heated in a microwave oven at 300 , 500 and $700 \mathrm{~W}$ for $5 \mathrm{~min}$ each. Primary mouse monoclonal anti-human TGF- $\beta$ (NCL-TGF- $\beta$; Novocastra; Leica Microsystems, Inc., Buffalo Grove, IL, USA) and rabbit polyclonal antibody against VEGF-A (071420; EMD Millipore, Billerica, MA, USA) were used at 1:100 dilution. Specimens were incubated with antibodies overnight at $4^{\circ} \mathrm{C}$. Anti-Rabbit or Anti-Mouse HRP-DAB Cell \& Tissue Staining kits (CTS006 and CTS002, respectively; R\&D Systems, Inc., Minneapolis, MN, USA) were used for blocking non-specific binding, and antibody detection and visualization. The sections were subsequently counterstained with Mayer's hematoxylin. Omission of the primary antibody was used as the negative control. Staining of collagen fibers using Van Gieson's method was performed to identify the level of newly formed collagen in granulation tissue. Stained specimens were viewed under a light microscope (Olympus BX41; Olympus Corporation, Tokyo, Japan), and random areas were captured in high-powered images at a magnification of $x 400$. The number of capillaries in 9 representative fields were counted for each specimen, and the total number for each field was averaged.

VEGF-A and TGF- $\beta$ staining was semiquantitatively scored as follows: No staining, $-;$; weak staining, + ; intermediate staining, ++; and strong staining, +++. For statistical analysis, 
Table I. Baseline characteristic of the patients from the present study.

\begin{tabular}{|c|c|c|}
\hline Characteristics & Patients from $2007-2009^{a}$ & Patients from $2010-2012^{b}$ \\
\hline Patients, $\mathrm{n}$ & 15 & 12 \\
\hline Age range, years & $49-77$ & $47-69$ \\
\hline Age, mean \pm SEM & $59.38 \pm 1.37$ & $57.16 \pm 1.90$ \\
\hline Sex, male/female & $11 / 4$ & $10 / 2$ \\
\hline $\mathrm{BMI}<17$ at the time of esophagectomy, $\mathrm{n}$ & 9 & 7 \\
\hline $\mathrm{BMI}<17$ at the time of reconstruction, $\mathrm{n}$ & 6 & 3 \\
\hline Chemoradiation, $\mathrm{n}$ & 7 & 4 \\
\hline \multicolumn{3}{|l|}{ Location of the tumor within the esophagus, $n$} \\
\hline Upper & 4 & 2 \\
\hline Middle & 7 & 6 \\
\hline Lower & 4 & 4 \\
\hline \multicolumn{3}{|l|}{ Postoperative $\mathrm{T}$ feature, $\mathrm{n}$} \\
\hline $\mathrm{T} 2$ & 5 & 5 \\
\hline $\mathrm{T} 3$ & 10 & 7 \\
\hline Size of leak, $n$ & 2 & 2 \\
\hline$<1 \mathrm{~cm}$ & 1 & 1 \\
\hline $1-1.5 \mathrm{~cm}$ & 1 & 1 \\
\hline Conduit necrosis, n (\%) & $1(6.6)$ & $0(0.0)$ \\
\hline
\end{tabular}

aPatients that underwent surgery between 2007 and 2009, and were evaluated retrospectively; bPatients that underwent surgery between 2010 and 2012 whose esophageal samples were evaluated immunohistochemically. SEM, standard error of the mean; BMI, body mass index in the group.

the values of $0,1,2$ and 3 were respectively assigned to the intensity of staining. Collagen formation was analyzed using ImageJ version $1.45 \mathrm{j}$ software (National Institutes of Health, Bethesda, MD, USA). The granulation index was calculated as the percentage of collagen mapped to the whole estimated surface area.

Statistical analysis. Statistical analysis was performed with Statistica 10 software (StatSoft, Inc., Tulsa, OK, USA). Descriptive statistics, including mean and standard error, were used to summarize the data. Student's $t$-test was used for the analysis of independent variables. $\mathrm{P}<0.05$ was considered to indicate a statistically significant difference.

\section{Results}

Patients characteristics. A total of 27 patients that underwent esophagectomy between January 2007 and December 2012 were initially considered for the study. Two patients over the age of 75 were excluded due to postoperative mortality; each succumbed following esophagoplasty (reconstructive second operation), with 1 mortality caused by complications resulting from necrosis of a colonic replacement for the esophagus and the other as a result of cardiac failure. Out of 25 patients, anastomotic leakage was observed in 4 and the mean leakage duration was 13 days. Out of 12 patients who underwent esophagectomy and secondary reconstruction between January 2010 and December 2012 (the subjects of immunohistochemical examination), 2 patients developed fistulas. Leakage occurred only if the esophagus was anastomosed with the colon. There were no leaks following ileo-coloplasty, where the esophagus was anastomosed with the ileal portion of the substitute. In these 2 cases, slight circumferential necrosis of the substitute caused the leak. There were no significant differences between microvessel density and levels of proangiogenic factors among the patients who developed leakage in comparison with those who healed without complication. In this group of patients, the mean time of leakage healing was 12 days There were no postoperative mortalities in this group.

The interval between esophagectomy and reconstruction varied based on the period of oncological treatment, organization of oncological therapy, the institution's schedule and the patient's attitude towards therapy. The shortest period was 2-3 months in the majority of patients, while the longest was 6 months in 1 patient and 5 months in 3 patients. Two patients postponed reconstruction for subjective reasons and fear of surgery, despite having completed radiochemotherapy for up to 3.6 months, while a further 2 patients underwent postponed reconstruction for objective reasons, including organization of oncological care.

Leakages were successfully treated conservatively. Mediastinitis occurred in only 1 patient who experienced necrosis of the conduit. During the treatment, patients were fed by gastrostomy.

Immunohistochemistry. Results of statistical analysis are presented in Table II. At the time of reconstructive surgery, a statistically significant increase was observed in microvessel 
Table II. Statistical analysis of VEGF-A, TGF- $\beta$ and collagen staining intensity, and number of vessels in the esophageal tissue at the time of the esophagectomy (NET) and during reconstructive surgery (GET).

\begin{tabular}{lccr}
\hline Characteristic & Mean & SEM & P-value $^{\mathrm{a}}$ \\
\hline NET & & & \\
TGF- $\beta$ & 1.088 & 0.077 & \\
VEGF-A & 0.000 & 0.000 & \\
Collagen area, $\%$ & 5.188 & 1.011 & 0.001 \\
Vessels, $n$ (range) & $19.70(10-36)$ & & 0.001 \\
GET directly prior to anastomosis construction & & 0.118 & 0.028 \\
TGF- $\beta$ & 2.094 & 0.212 & 0.028 \\
VEGF-A & 1.325 & 6.205 & 0.029 \\
Collagen area, $\%$ & 38.372 & $23.19(16-37)$ & \\
Vessels, $n$ (range) & & & \\
\hline
\end{tabular}

aP-values correspond to NET vs. GET. VEGF, vascular endothelial growth factor; TGF, transforming growth factor; NET, normal esophageal tissue; GET, granulation esophageal tissue; SEM, standard error of the mean.

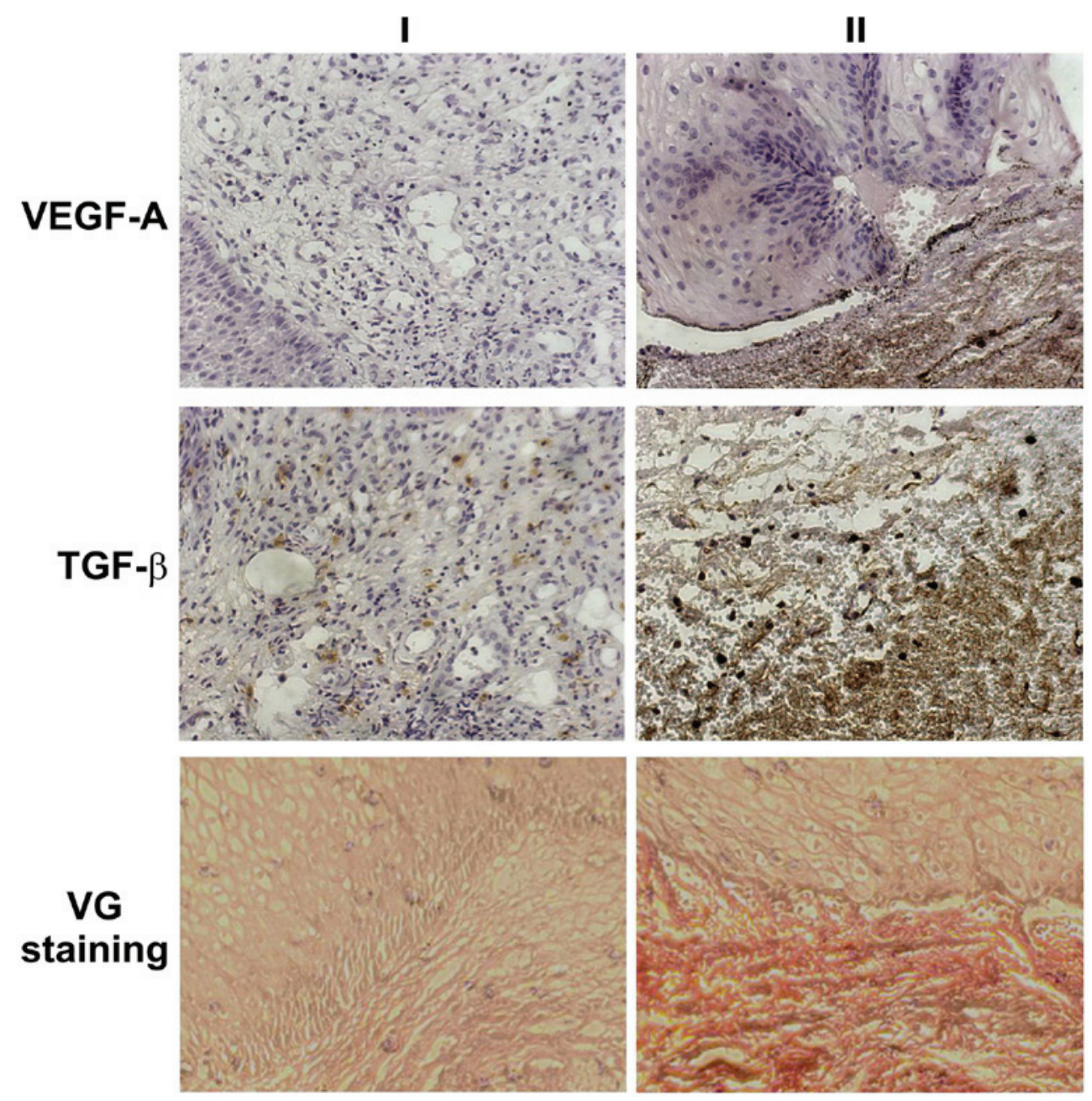

Figure 1. Representative microscopy images of select immunohistochemical staining of VEGF-A, TGF- $\beta$ and VG collagen dying. Absence of VEGF-A in normal esophageal tissue (I) at the time of esophagectomy and its high expression in granulation tissue (II) during reconstructive surgery. Level of TGF- $\beta$ and collagen staining in granulation tissue (II) relative to normal tissue (I). VEGF, vascular endothelial growth factor; TGF, transofmring growth factor; VG, Van Gieson's.

density in all esophageal specimens $(\mathrm{P}<0.03)$. A significant difference was also demonstrated in the immunohistochemical staining intensity of TGF- $\beta$, VEGF-A and collagen fibers $(\mathrm{P}<0.05)$. Fig. 1 presents the differences in immunohistochemical staining in selected microscopic images of the esophageal specimens at the time of esophagectomy and during reconstructive surgery. 


\section{Discussion}

In the Department of Gastrointestinal and General Surgery, Wrocław Medical University, the preferred method of surgical treatment for middle esophageal cancer extending between the azygos vein and the lower pulmonary vein is the two-stage procedure with esophageal reconstruction of the colon. In the present study, approximately two thirds of the patients were in the advanced stage of the disease, with significant dysphagia, and nearly half of them had a BMI of $<17$ at the time of esophagectomy. Nutritional status and nutritional support, particularly by way of patients undergoing esophagectomy, are among factors that improve perioperative mortality rate and survival (23). Patients are at a higher risk of complications following coloplasty $(8,24)$. Advanced age and prolonged duration of surgery together with diabetes, renal failure and poor general condition are reported to be important risk factors responsible for perioperative mortality following esophagectomy $(8,9)$. In the current study, there were no mortalities subsequent to esophagectomy. Two patients succumbed to complications following the second surgery. Reconstruction of the esophagus (mortality rate, $7.4 \%$ ) remains at an average level in comparison with high volume institutions that report a mortality rate $<5 \%$, although patient selection and a small study sample appear to bias the outcomes. The leak rate tends to be higher if the colon conduit rather than gastric tube is used, and when it is placed in the anterior mediastinum rather than the posterior mediastinal route (1). Patients who succumb to complications following reconstruction surgery are typically of an advanced age, and necrosis of the esophageal replacement is a complication with an extremely high mortality rate (23-25). In the series of patients who were operated between January 2010 and December 2012, the incidence of mortality subsequent to coloplasty was $0 \%$. There was no statistically significant difference between these 2 groups in terms of as to patients age, gender, BMI or tumor size according to the tumornode-metastasis classification.

Using the two-stage approach and careful patient selection for coloplasty, the patients of the present study achieved low in-hospital mortality and a low reoperation rate. Right thoracotomy is often favored to left thoracotomy as it offers better approach to the esophagus and enveloping lymph nodes. In approximately half of esophageal cancer cases, the disease develops in the middle esophagus $(1,8,9,24,25)$; therefore, the optimal treatment is subtotal esophagectomy. Intrathoracic anastomosis performed in mid-esophageal lesions is not free of the risk of cancer-positive resections margins (25). Certain studies have emphasized that R0 resection in particular has an impact on survival rate (26). None of the patients in the present study that underwent anastomosis on the neck after subtotal esophagectomy were positive for cancer within the resection margin. One-stage esophagectomy and coloplasty is a long surgery with a mean duration of $7.5 \mathrm{~h}$, and has a higher mortality rate compared with the two-stage procedure $(1,8,9,24)$.

Furthermore, prolonged surgery duration causing greater surgical stress is associated with acute lung injury, which leads to systemic inflammatory response syndrome and other critical complications, including anastomotic leakage (9).
Leakage of the cervical anastomosis remains the most serious complication of esophageal reconstructive surgery $(1,3,8,9)$.

The concept of a postponed anastomosis of the cervical esophagus with the intestine provides an advantage for the esophageal stump to accumulate growth factors and various molecules supporting its recovery. The present study observed a significant increase in microvessel density in all esophageal biopsies acquired at the time of reconstructive surgery compared with probes taken during esophagectomy $(\mathrm{P}<0.03)$, which was associated with significant differences in immunohistochemical staining intensity. The current study focused on the expression of VEGF-A and TGF- $\beta$, which are responsible for angiogenesis and wound healing (10,13-15). VEGF-A is an obvious choice, since is the key marker of angiogenesis.. Besides functioning in the induction of angiogenesis, TGF- $\beta$ also possesses anti-inflammatory properties and exhibits an inhibitory effect on collagenase activity, thus protecting newly synthesized collagen within anastomoses (17). Bernstein et al (27) reported that topical application of TGF- $\beta$ improves radiation-impaired wounds. Transposition of the esophageal stump to the anterior mediastinum aids the avoidance of mediastinum contamination when leakage occurs; it also allows for conservative treatment of the leakage, resulting in a shorter hospital stay.

Patients of the present study qualified for conservative treatment following the analysis of clinical data and, if necessary, endoscopy was performed to evaluate the extent of ischemia of the anastomotic circumference. The current study reported that fistulas developed in $16 \%$ of cases; data from the literature indicates that this complication may develop in $3-40 \%$ of cases (2,28-33). However, actual data regarding leak rates may be underestimated by defining leakage as a condition requiring use of surgical intervention, as one third of leaks in the chest do not require surgery $(7,31)$. The incidence of this complication varies among studies. Anastomosis healing may be affected not only by the conduit type and location of anastomosis, but also by the preoperative status of the patient. Furthermore, according to the literature, leakage rate following colo-esophagoplasty, including patients with caustic strictures, remains higher than the leakage rate following reconstruction with a gastric tube, and ranges from $6.7-46.4 \%(1,24,28)$. In the present patients, leakage occurred only if the esophagus was anastomosed with the colon. There was no leak among patients who had the esophagus anastomosed with the ileum. Leakage incidence is identified to be higher in neck anastomoses compared with intrathoracic anastomoses $(1,24,29)$. The present study only included patients with esophageal cancer who underwent two-stage surgery; the esophagus was anastomosed with the ileum (ileo-coloplasty) or the colon (coloplasty). The present study was not large, but the results were homogeneous. All reconstructions were performed on the right hemi-colon, at the neck and in a squamous cell carcinoma patient population. Similar to other studies, the current study lacked a control group; thus, these results may only be compared with published data $(1,24,28,30-32)$.

The basic rules and phases of the intestinal wound healing process are similar to the healing process of skin wounds. However, there are key differences in the slowly progressing proliferation and remodeling phase. While mucosal resurfacing 
occurs rapidly and is completed after 7 days, the proliferation and overlapping remodeling phase lasts for months (34). The results of the current study confirmed this, as all esophageal specimens obtained at the time of reconstruction had a significantly larger vascular network, a high granulation index and the growth factors essential for the proliferation and early remodeling phase.

Rijcken et al (34) reported that recovery of the vascular network and increased angiogenesis serve an essential role in the process of anastomosis healing. In the present study, all esophageal specimens obtained following esophagectomy exhibited lower levels of VEGF-A and TGF- $\beta$ compared with those obtained at the time of esophageal anastomosis formation. VEGF-A and TGF- $\beta$ levels were associated with vascular net density and were statistically higher in esophageal stumps at the time of reconstruction, although the vascular network of the esophageal stump may be damaged while being translocated to the anterior mediastinum during the first surgical stage.

The current study also noted that the new vessel network and existing key growth factors overlapped with the further proliferative phase of wound healing and promoted a favorable environment for healing anastomoses constructed up to several months later. This finding is in agreement with Ishii et al (35), who reported that following VEGF-A application, microangiographic analysis of biopsies of colonic anastomoses in rats revealed significantly higher capillary counts and granulation tissue formation in comparison with saline-treated controls (35-37). The present study demonstrated that growth factors from the esophageal stump were physiologically present and anastomosis healing took place in a naturally-created environment. We hypothesize that in two-stage reconstruction, the ischemic tissue is under direct influence of growth factors already synthesized and delivered by the esophageal tissue that promote its vascularization. Therefore, in such an environment, even if the terminal circumference of the colonic transplant suffers from ischemia and esophago-intestinal fistula occurs, the tissue heals more easily. The mean time of fistula healing in the current study was 13 days, and none of the patients who developed the leak required any surgical intervention. All stumps were in their proliferative phase of wound healing, which indicates that proangiogenic VEGF-A and TGF- $\beta$ were already highly expressed in the anastomosed tissue and did not require delivery to the healing site. A number of clinical trials have failed to successfully complete wound healing following topical application of exogenous VEGF (37-39).

The current study has presented a novel surgical approach for the performance of difficult anastomoses. In general, tension-free suturing and excellent blood perfusion lead to primary spontaneous healing of anastomoses $(2-5,36)$. In pathological conditions, including systemic or local severe inflammation and ischemia, the healing process may be impaired and result in the occurrence of fistulas or fibrosis, subsequently leading to stenosis of the site of the anastomosis. In such conditions, anastomosis may be postponed and performed in a second surgery. Postponed esophageal anastomosis has an obvious disadvantage; the prolonged time of feeding omitting the natural way of oral food intake. Nevertheless, it may be reserved for select patients, particularly in the era of neoadjuvant chemoradiation.
In conclusion, two-staged esophagectomy enables high-risk patients to recover from surgical stress after esophagectomy and to avoid critical complications. Postponed cervical anastomosis conducted with the colon replacement is not free from leak occurrence; however, the esophageal stump, which accumulated proangiogenic factors and acquired novel microvessel networks, heals more easily.

\section{References}

1. Urschel JD, Urschel DM, Miller JD, Bennett WF and Young JE: A meta-analysis of randomized controlled trials of route of reconstruction after esophagectomy for cancer. Am J Surg 182: 470-475, 2001.

2. Feith M, Gillen S, Schuster T, Theisen J, Friess H and Gertler R: Healing occurs in most patients that receive endoscopic stents for anastomotic leakage; dislocation remains a problem. Clin Gastroenterol Hepatol 9: 202-210, 2011.

3. Schubert D, Dalicho S, Flohr L, Benedix F and Lippert H: Management of postoperative complications following esophagectomy. Chirurg 83: 712-718, 2012 (In German).

4. Böhm G, Mossdorf A, Klink C, Klinge U, Jansen M, Schumpelick V and Truong S: Treatment algorithm for postoperative upper gastrointestinal fistulas and leaks using combined vicryl plug and fibrin glue. Endoscopy 42: 599-602, 2010.

5. Reavis KM: The esophageal anastomosis: How improving blood supply affects leak rate. J Gastrointest Surg 13: 1558-1560, 2009.

6. Sarela AI, Tolan DJ, Harris K, Dexter SP and Sue-Ling HM: Anastomotic leakage after esophagectomy for cancer: A mortality-free experience. J Am Coll Surg 206: 516-523, 2008.

7. Cools-Lartigue J, Andalib A, Abo-Alsaud A, Gowing S, Nguyen M, Mulder D and Ferri L: Routine contrast esophagram has minimal impact on the postoperative management of patients undergoing esophagectomy for esophageal cancer. Ann Surg Oncol 21: 2573-2579, 2014.

8. Sueyoshi S, Yamana H, Fujita H, Tanaka T, Toh U, Kubota M, Tanaka Y, Mine T, Sasahara H and Shirouzu K: Radical esophagectomy and secondary anastomosis for high-risk patients with intrathoracic esophageal carcinoma. Jpn J Thorac Cardiovasc Surg 48: 683-687, 2000.

9. Morita M, Nakanoko T, Kubo N, Fujinaka Y, Ikeda K, Egashira A, Saeki H, Uchiyama H, Ohga T, Kakeji Y, et al: Two-staged operation for high-risk patients with thoracic esophageal cancer: An old operation revisited. Ann Surg Oncol 18: 2613-2621, 2011.

10. Eming SA, Brachvogel B, Odorisio T and Koch M: Regulation of angiogenesis: Wound healing as a model. Prog Histochem Cytochem 42: 115-170, 2007.

11. Sharma RA, Harris AL, Dalgleish AG, Steward WP and O'Byrne KJ: Angiogenesis as a biomarker and target in cancer chemoprevention. Lancet Oncol 2: 726-732, 2001.

12. Yazdani S, Kasajima A, Tamaki K, Nakamura Y, Fujishima F, Ohtsuka H, Motoi F, Unno M, Watanabe M, Sato Y and Sasano H: Angiogenesis and vascular maturation in neuroendocrine tumors. Hum Pathol 45: 866-874, 2014.

13. Bao P, Kodra A, Tomic-Canic M, Golinko MS, Ehrlich HP and Brem H: The role of vascular endothelial growth factor in wound healing. J Surg Res 153: 347-358, 2009.

14. Kim IY, Kim MM and Kim SJ: Transforming growth factor-beta: Biology and clinical relevance. J Biochem Mol Biol 38: 1-8, 2005.

15. Wietecha MS and DiPietro LA: Therapeutic approaches to the regulation of wound angiogenesis. Adv Wound Care (New Rochelle) 2: 81-86, 2013.

16. Reinke JM and Sorg H: Wound repair and regeneration. Eur Surg Res 49: 35-43, 2012.

17. Eming SA and Hubbell JA: Extracellular matrix in angiogenesis: Dynamic structures with translational potential. Exp Dermatol 20: 605-613, 2011.

18. Schultz GS and Wysocki A: Interactions between extracellular matrix and growth factors in wound healing. Wound Repair Regen 17: 153-162, 2009.

19. Pakyari M, Farrokhi A, Maharlooei MK and Ghahary A: Critical role of transforming growth factor beta in different phases of wound healing. Adv Wound Care (New Rochelle) 2: 215-224, 2013.

20. Egginton S and Gaffney E: Tissue capillary supply-it's quality not quantity that counts! Exp Physiol 95: 971-979, 2010. 
21. Wietecha MS, Cerny WL and DiPietro LA: Mechanisms of vessel regression: Toward an understanding of the resolution of angiogenesis. Curr Top Microbiol Immunol 367: 3-32, 2013.

22. Tirziu D and Simons M: Endothelium as master regulator of organ development and growth. Vascul Pharmacol 50: 1-7, 2009.

23. Llop-Talaveron JM, Farran-Teixidor L, Badia-Tahull MB, Virgili-Casas M, Leiva-Badosa E, Galán-Guzmán MC, Miró-Martin M and Aranda-Danso H: Artificial nutritional support in cancer patients after esophagectomy: 11 years of experience. Nutr Cancer 66: 1038-1046, 2014.

24. Hamai Y, Hihara J, Emi M, Aoki Y and Okada M: Esophageal reconstruction using the terminal ileum and right colon in esophageal cancer surgery: Surg Today 42: 342-350, 2012.

25. Huang HT, Wang F, Shen L, Xia CQ, Lu CX and Zhong CJ: Clinical outcome of middle thoracic esophageal cancer with intrathoracic or cervical anastomosis. Thorac Cardiovasc Surg 63: 328-334, 2015

26. McK Manson J and Beasley WD: A personal perspective on controversies in the surgical management of oesophageal cancer. Ann R Coll Surg Engl 96: 575-578, 2014.

27. Bernstein EF, Harisiadis L, Salomon G, Norton J, Sollberg S, Uitto J, Glatstein E, Glass J, Talbot T, Russo A, et al: Transforming growth factor-beta improves healing of radiation-impaired wounds. J Invest Dermatol 97: 430-434, 1991.

28. Markar SR, Arya S, Karthikesalingam A and Hanna GB Technical factors that affect anastomotic integrity following esophagectomy: Systemic review and meta-analysis. Ann Surg Oncol 20: 4274-4281, 2013.

29. Yasuda T and Shiozaki H: Esophageal reconstruction with colon tissue. Surg Today 41: 745-753, 2011.

30. Paul S and Altorki NJ: Outcomes in the management of esophageal cancer. Surg Oncol 110: 599-610, 2014.
31. Saeki H, Morita M, Tsuda Y, Hidaka G, Kasagi Y, Kawano H, Otsu H, Ando K, Kimura Y, Oki E, et al: Multimodal treatment strategy for clinical T3 thoracic esophageal cancer. Ann Surg Oncol 20: 4267-4273, 2013.

32. Morita M, Otsu H, Kawano H, Kumashiro R, Taketani K, Kimura Y, Saeki H, Ando K, Ida S, Oki E, et al: Advances in esophageal surgery in elderly patients with thoracic esophageal cancer. Anticancer Res 33: 1641-1647, 2013.

33. Schaible A, Sauer P, Hartwig W, Hackert T, Hinz U, Radeleff B, Büchler MW and Werner J: Radiologic versus endoscopic evaluation of the conduit after esophageal resection: A prospective, blinded, intraindividually controlled diagnostic study. Surg Endosc 28: 2078-2085, 2014

34. Rijcken E, Sachs L, Fuchs T, Spiegel HU and Neumann PA: Growth factors and gastrointestinal anastomotic healing. J Surg Res 187: 202-210, 2014

35. Ishii M, Tanaka E, Imaizumi T, Sugio Y, Sekka T, Tanaka M, Yasuda M, Fukuyama N, Shinozaki Y, Hyodo K, et al: Local VEGF administration enhances healing of colonic anastomoses in a rabbit model. Eur Surg Res 42: 249-257, 2009.

36. Eming SA, Krieg T and Davidson JM: Inflammation in wound repair: Molecular and cellular mechanisms. J Invest Dermatol 127: 514-525, 2007.

37. Brudno Y, Ennett-Shepard AB, Chen RR, Aizenberg M and Mooney DJ: Enhancing microvascular formation and vessel maturation through temporal control over multiple pro-angiogenic and pro-maturation factors. Biomaterials 34: 9201-9209, 2013.

38. Barrientos S, Brem H, Stojadinovic O and Tomic-Canic M: Clinical application of growth factors and cytokines in wound healing. Wound Repair Regen 22: 569-578, 2014.

39. Grommes J, Binnebösel M, Klink CD, von Trotha KT, Schleimer K, Jacobs MJ, Neumann UP and Krones CJ: Comparison of intestinal microcirculation and wound healing in a rat model. J Invest Surg 26: 46-52, 2013. 